\title{
Herméneutique du symbole et herméneutique du soi : Paul Ricœur et la logique du double sens
}

\section{Camille Riquier}

\section{(2) OpenEdition}

1 Journals

Édition électronique

URL : http://journals.openedition.org/alter/1419

DOI : $10.4000 /$ alter. 1419

ISSN : 2558-7927

Éditeur :

Association ALTER, Archives Husserl (CNRS-UMR 8547)

\section{Édition imprimée}

Date de publication : 1 octobre 2011

Pagination : 181-197

ISBN : 978-2-9522374-7-5

ISSN : $1249-8947$

\section{Référence électronique}

Camille Riquier, «Herméneutique du symbole et herméneutique du soi : Paul Ricœur et la logique du double sens », Alter [En ligne], 19 | 2011, mis en ligne le 01 janvier 2020, consulté le 23 janvier 2020. URL : http://journals.openedition.org/alter/1419; DOI : 10.4000/alter.1419 


\section{HERMÉNEUTIQUE DU SYMBOLE ET HERMÉNEUTIQUE DU SOI : PAUL RICEUR ET LA LOGIQUE DU DOUBLE SENS}

Camille Riquier

Ne sachant pas écouter, ils ne savent pas non plus parler (Héraclite)1.

Le langage n'a pas toujours été l'énigme qu'il est devenu pour la pensée occidentale. Il a fallu que la philosophie s'éloigne de la simplicité de sa source grecque. L'incapacité à traduire le terme grec sans le diffracter en une multitude d'approximations - langage, discours, parole, etc. - montre à elle seule l'étendue de la distance qui

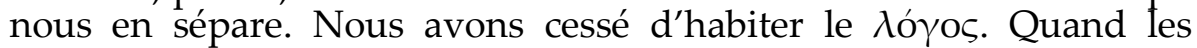
Grecs avaient une confiance absolue dans le langage, qui ne pouvait pas ne pas dire l'être et se reverser aussitôt dans le monde qu'il nommait, notre modernité a rendu le médium opaque, et l'interroger comme un objet ainsi que nous le faisons trahit la défiance que nous lui portons. On peut se demander si la philosophie, qu'on qualifie aujourd'hui si étrangement de "continentale » depuis l'autre rive de l'Atlantique, n'apparaît pas déjà au loin comme une «Atlantide » sur le point d'être engloutie, qu'on ne voit presque plus, qu'on n'entend presque plus. Sans l'avoir voulu, en la désignant ainsi, la philosophie analytique dit pourtant une vérité sur notre situation à tous. Car ce qu'elle comprend d'un point de vue géographique doit se comprendre aussi d'un point de vue historique, pour la vieille Europe : nous

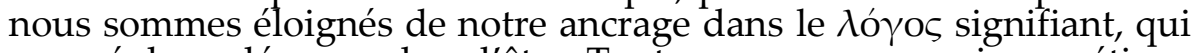
a cessé de se déverser dans l'être. Tout se passe comme si nous étions exclus du langage et que notre menace à tous, circulant parmi ses décombres, était de ne plus rien entendre, de manipuler des signes vides là où nous n'arrivons plus à écouter, et de ressembler à cet idiot qui regarde le doigt, quand le sage lui montre la lune.

1. Héraclite, fragment B 19, tr. fr. M. Conche, Paris, PUF, « Epiméthée », p. 20. 
C'est ainsi que le langage est devenu, pour nous modernes, « un problème de culture $»^{2}:$ " le monde technologique dans lequel nous vivons » est traversé par un projet de maîtrise qui comprime le temps, efface nos mémoires et finit par nous rendre sourds aux paroles déjà dites. Au lieu d'accueillir la parole qui nous précède toujours déjà, nous voulons nous en rendre maître et nous placer désormais à son point d'origine sans cesse recommencé. La force dispensatrice du $\lambda$ ó $\mathrm{o}$ semble s'être peu à peu dissipée pour passer tout entière dans la forme étale qui l'a mise sous nos yeux, et qui a fait du langage, ainsi vidé de son contenu, un instrument de plus, soumis à notre discrétion. Par là même, notre langage s'est progressivement disloqué à mesure que nous le rendions "plus précis, plus univoque, plus technique en un mot $»^{3}$ en vue d'un usage de plus en plus varié, dans nos arts et nos sciences. Plus encore, $c^{\prime}$ est en cherchant à surmonter sa dispersion que les nouvelles disciplines qui prirent explicitement le langage pour thème de leur investigation l'ont en fait précipitée et révélée par leur nombre même : philosophie analytique, philologie, exégèse biblique, structuralisme, psychanalyse, phénoménologie, etc.

Bref, « l'unité du parler humain fait aujourd'hui problème. Tel est $l^{\prime}$ horizon le plus vaste sur lequel se découpe [la] recherche $»^{4}$ de Paul Ricœur et à quoi il aspire : "une grande philosophie du langage " $^{5}$, $\mathrm{du}$ «langage intégral ». Telle est aussi la situation historique où nous a jetés notre modernité, en faisant de nous des tard-venus, après que tout a été dit. Nul doute que la question du langage est d'abord ce qui doit nous remettre en chemin vers lui, comme l'affirmait déjà Heidegger. Toutefois, le problème une fois posé, deux attitudes, au moins, sont possibles pour y répondre, qui sont deux manières antithétiques de remembrer le langage, dont aucune d'ailleurs ne renie notre modernité. Ou bien, souhaitant ne pas vivre cette distanciation comme une perte et une crise de notre culture, on se propose $d^{\prime}$ accuser plus encore la distance, en décidant d'appréhender le langage du dehors et de l'évider comme un objet, qu'on détache en amont du sujet qui l'énonce, en aval du monde qu'il énonce. Il devient alors un transcendantal ; tel est le choix d'un certain nombre de philosophies du langage. Ou bien on affirme à nouveau notre appartenance au langage, en dépit de la distance, en s'inscrivant à nouveau en lui, dans son plein, dans l'espoir de le restaurer. Mais alors il faut renon-

\footnotetext{
2. P. Ricœur, «Prévision économique et choix éthique », dans Histoire et Vérité (cité HV), 1955, Paris, Seuil, 2001, p. 355.

3. P. Ricœur, «Herméneutique des symboles et réflexion philosophique (I)», dans Le Conflit des interprétations. Essais d'herméneutique (cité C), Paris, Seuil, 1969, p. 284 ; Philosophie de la volonté, t. II (cité Ph.V. II), (1960), Paris, Seuil, 2009, p. 568.

${ }^{4}$. P. Ricœur, De l'interprétation. Essai sur Freud (cité E), (1965), Paris, Seuil, 1995, p. 14.

5. Ibid., p. 13.
} 
cer à le dominer. Car c'est quand le langage nous échappe qu'il peut dire et nous interpeller, et remplir à nouveau sa "fonction ontologique ». Il redevient un milieu; tel est le choix de l'herméneutique. "Ainsi c'est la même époque qui développe la possibilité de vider le langage et celle de le remplir à nouveau $»^{6}$. Bref, soit elle le maîtrise de l'extérieur et cherche à constituer un langage univoque, débarrassé de ses ambiguïtés naturelles et entièrement soumis à une technique qui le formalise. Le langage fera l'objet d'une science. Soit elle l'investit à nouveau de l'interieur, et consent à son équivocité en se pliant à sa logique du double sens. Le langage est le milieu où circulera l'art de l'herméneute.

Ce langage plein, qui nous lie par son double sens, voire ses multiples sens, qu'il s'agit moins de dire que d'écouter à l'aide d'une herméneutique, c'est ce que Ricœur appelle le langage symbolique qui l'occupe dans toute la première partie de son ouvre, jusqu'en 1970. Rencontrant la notion de symbole dès son étude sur Jaspers ${ }^{7}$, il la déploie de façon centrale dans le second volet de sa Philosophie de la volonté qui l'invite à se concentrer autour des symboles du mal. Il poursuit ensuite son étude dans son Essai sur Freud en l'élargissant à d'autres ensembles symboliques, comme ceux qu'offre la psychanalyse. Dans Le Conflit des interprétations, il s'ouvre enfin à la structure $\mathrm{du}$ symbole "en tant que structure langagière spécifique ${ }^{8}$. S'il descend ainsi d'un exemple particulier de symbole en direction de sa structure générale, en régressant de plus en plus vers la méthode herméneutique qui la sous-tend, sa reflexion garde tout au long un souci épistémologique, présent dès le début, à l'occasion des premiers symboles choisis. Quelle définition en donne-t-il? "Selon nous le symbole est une expression linguistique à double sens qui requiert une interprétation » puisqu'il "veut dire autre chose que ce qu'il dit $»{ }^{9}$. En effet, sitôt qu'une parole est dite à une époque et reçue dans une autre, sa signification prend un tour irréductiblement équivoque, voire multivoque, par la distance même qu'elle a franchie entretemps. Elle se déphase en un sens littéral, lié au contexte historique qui fut celui de son énonciation, et un sens spirituel, fruit du travail d'interprétation, qui surmonte l'éloignement culturel et l'incorpore «à la compréhension présente qu'un homme peut prendre de luimême $»^{10}$. Autrement dit, le double sens appartient voire se confond

\footnotetext{
6. P. Ricœur, C., p. 284 ; Ph.V. II, p. 568.

7. M. Dufrenne et P. Ricœur, Karl Jaspers et la philosophie de l'existence, Paris, Seuil, 1949, p. 291 : «[...] dans le symbole l'objet disparaît comme objet, tandis que la Transcendance apparaît en lui, sans qu'on puisse séparer le symbole de l'être qu'il signifie, ni faire une exégèse en clair du chiffre à la façon du déchiffrement d'un langage codé ».

8. P. Ricœur, "Herméneutique et symbolisme" (1988) dans Écrits et conférences, II, Paris, Seuil, 2010, p. 28.

9. P. Ricœur, E., respectivement p. 19, 17.

10. P. Ricœur, « Existence et herméneutique », C., p. 8.
} 
avec le champ herméneutique lui-même, en lui donnant sa définition minimale, avant tous les conflits qui ne manqueront pas de s'y dérouler entre interprétations rivales.

Par ailleurs, s'il arrive à Ricœur de définir le champ herméneutique comme étant la région du double sens, découpée dans le champ plus large du langage, il ne peut laisser coexister l'un à côté de l'autre deux types de langage aussi différents que le langage univoque et le langage équivoque, que par une "étrange homonymie " le symbole, remarque-t-il, a servi à désigner tous deux : "logique symbolique » d'un côté, "herméneutique des symboles » de l'autre. Ici, pourtant, nulle transaction possible: ou bien le langage est univoque, et l'équivocité n'est qu'une ambiguïté qu'il faut dissiper; ou bien le langage est équivoque, et l'exigence d'univocité ne sera jamais qu'un vain souhait auquel il faut renoncer. Autrement dit, le double sens doit posséder lui aussi une logique qui lui est propre en puisant son $a$ priori dans la réflexion même. Parce que la «logique symbolique » ne réunifie pas le langage sans exclure, avec l'équivoque, rien de moins que le langage naturel, la logique du double sens doit être "d'ordre transcendantal » si elle veut être incontestable et renvoyer le langage univoque au rêve de quelques philosophes. Son domaine de validité objective doit ainsi trouver son assise au sein même de la subjectivité, «dans le mouvement d'appropriation de soi par soi qui constitue l'activité réflexive ${ }^{11}$.

Aussi devons-nous rencontrer la logique du double sens à trois niveaux si le symbole veut être fondé véritablement : réflexif, phénoménologique et herméneutique. Telle est d'ailleurs la triple tradition philosophique dont Ricour se réclame. Cette tradition, écrit-il, " est dans la ligne d'une philosophie réflexive; elle demeure dans la mouvance de la phénoménologie husserlienne; elle veut être une variante herméneutique de cette phénoménologie $»^{12}$. Loin qu'il faille voir dans son parcours intellectuel une synthèse fortuite d'influences disparates, c'est dans le problème du symbole que toutes se nouent étroitement, en sorte que la logique du double sens doit pouvoir se récupérer comme structure réflexive et intentionnelle, avant de recevoir sa "greffe herméneutique ». C'est dire aussi que ces trois traditions $\mathrm{n}^{\prime}$ interviennent pas au même titre, mais épousent un dénivelé, qui a toujours préservé Ricœur de tout recours à l'éclectisme. L'herméneutique lui fournit le problème ; la phénoménologie, la méthode ; la philosophie réflexive, la doctrine. L'herméneutique lui fournit d'abord le probleme du sens. Mais par là même, elle doit renoncer à l'idée, qui fut celle de Dilthey, de tirer d'elle seule « une méthode digne de lutter

11. P. Ricœur, E., p. 62-63.

12. P. Ricœur, «De l'interprétation» (1983) dans Du texte à l'action. Essais d'herméneutique (cité

TA), Paris, Seuil, 1986, p. 29. 
à armes égales avec celles des sciences de la nature ${ }^{13}$. Aussi doit-elle emprunter sa méthode à la phénoménologie. Enfin, parce qu'elle ne prétend pas au titre de science, la phénoménologie herméneutique, à son tour, ne peut s'élever à sa dimension philosophique qu'en s'orientant vers la doctrine du cogito, dont elle est la relance ("variante»): en surmontant ce qui nous éloigne d'une époque culturelle révolue, la compréhension des signes ne devient en toute rigueur philosophique que si elle sert en même temps à "la compréhension de soi ». En transplantant ainsi le problème du double sens sur une méthode phénoménologique et une doctrine réflexive, il sera bien question d'une double greffe herméneutique, et non pas d'une seule ${ }^{14}$.

Enfin, ces trois traditions ne s'entrelacent pas sans rencontrer, à chaque fois qu'elles se croisent, l'adversaire irréductible de Ricœur, qui fut d'abord celui de son maître, Gabriel Marcel : l'idéalisme. Il est faux de dire que Ricœur n'avait pas d'ennemi. Toutefois, il le savait intérieur et partout présent comme une tentation qui pèse sur chacune des traditions evoquées, avec pour principal effet de les rendre incompatibles entre elles. En raison de sa prétention totalisante, l'idéalisme interdit en effet toute collaboration et hiérarchisation de se mettre en place. Ricœur rencontre ainsi son adversaire à chacun des trois niveaux, qui empêche de les concilier : l'idéalisme réflexif, l'idéalisme phénoménologique, l'idéalisme linguistique. Si le cogito, pas plus que la phénoménologie, ne sont d'emblée herméneutiques, ce n'est pas qu'ils devaient recevoir l'herméneutique comme un corps qui leur fût étranger, mais parce qu'il leur est arrivé de succomber à la tentation idéaliste, qui escamota le dénivellement qui eût permis aux différentes traditions de coopérer entre elles, en leur octroyant le privilège, aussi séduisant qu'exorbitant, de fournir, chacune pour elle-même et à l'exclusion des autres, le problème, la méthode et la doctrine. Bref, la tradition réflexive, ou encore phénoménologique, sont herméneutiques en droit, mais non pas en fait. Aussi doiventelles être préalablement soumises à la critique, si l'on veut les débarrasser de leur métaphysique sous-jacente qui, en l'état, rejette le greffon herméneutique et empêche jusqu'à l'implantation de se faire. C'est en l'attachant fermement à la subjectivité que l'arc herméneutique pourra y reconduire au bout de sa trajectoire, ne la quittant que pour mieux la situer dans l'être, en la gonflant des symboles qu'elle aura recueillis hors d'elle au sein d'une réflexion enfin devenue concrète. Rappelant la condition langagière de toute expérience, Ricœur nous enjoint de porter au langage la conscience, qui a besoin d'être dite pour être pleinement vécue. Alors, nous pourrons rendre

\footnotetext{
13. P. Ricœur, « Existence et herméneutique », C., p. 11.

14. Comme le suggère le beau titre du livre de J. Greisch, Le Cogito herméneutique. L'herméneutique philosophique et l'héritage cartésien, Paris, Vrin, 2000.
} 
son plein sens à cette formule de Kant que Ricœur aime à citer : «Le symbole donne à penser ».

\section{La ligne réflexive : Descartes et Ricœur - le cogito replacé dans le plein du langage}

La voie que prolonge Ricœur demeure celle du cogito, qui brode ses figures historiques sur le canevas cartésien. En effet, « le Cogito de Descartes est seulement l'un des sommets [...] d'une chaîne de Cogito qui constituent la tradition réflexive $»^{15}$. Placer son cogito brisé «à égale distance » de Descartes et de Nietzsche, comme on l'a souvent fait, c'est sombrer dans un éclectisme stérile, qui nous semble moins être le péril de Ricœur que de celui qui le commente. Car c'est occulter l'opération critique que Ricœur introduit à l'intérieur de la tradition réflexive, à laquelle il n'a jamais cessé d'appartenir. Ricœur, comme Bergson avant lui, est cartésien. Et sa philosophie ne rejette pas le Cogito; elle l'expose à la contestation afin de l'extraire de l'époque métaphysique qui l'a vu naître et de l'envoyer dans la nôtre, où la crise qu'il connaît le lie désormais à la grande problématique du "langage $»^{16}$. Autrement dit, elle le prolonge en l'enchaînant à la longue suite de ses interprétations, invitant la philosophie réflexive " non à se maintenir identique à elle-même en repoussant les assauts de l'adversaire, mais à prendre appui sur lui, à faire couple avec ce qui la conteste le plus ». Tel est le premier postulat herméneutique, auquel Ricœur consent pour lui-même: puisqu'il faut bien naître quelque part, il doit trouver un point de départ dans sa situation, qui lui ouvre certes l'horizon indéfini des cultures mais depuis la perspective limitée qui est la sienne, à savoir la tradition réflexive dont il hérite. Son ambition fut ainsi de réinterpréter le cogito à son époque, c'est-à-dire en supprimant la métaphysique qui lui ètait sousjacente, à l'époque où Descartes le formula pour la première fois. Il $\mathrm{s}^{\prime}$ agit bien de relancer le cartésianisme, à travers les objections qui lui furent adressées, ne renaissant qu'après avoir passé le feu des critiques.

Mais nous l'avions mentionné, parce que l'adversaire est intérieur à la tradition réflexive, celle-ci s'est comme scindée en deux lignes divergentes, quoiqu'entremêlées d'abord en tant qu'elles trouvèrent l'une et l'autre leur point d'amorce dans Descartes : d'un côté, un courant idéaliste, qui a cédé à ce qui n'était qu'une tentation pour Descartes lui-même, se cristallisant chez Kant et se fixant dans une egologie avec Fichte et Husserl (du moins celui des Méditations carté-

15. P. Ricœur, «La question du sujet : le défi de la sémiologie », C., p. 233.

16. P. Ricœur, E, p. 47. 
siennes); parallèlement, un autre courant s'est poursuivi, moins souvent cité et dont la grande majorité de ses représentants sont français: libre de faire appel au sentiment, il va de Descartes à Ricœur, en passant par Pascal, Rousseau, Maine de Biran, Bergson, Gabriel Marcel, Jean Nabert, jusqu'à la phénoménologie française elle-même. L'un et l'autre courant ont repris le cogito dans son énoncé principiel : "je pense, je suis », mais en plaçant chacun l'accent à un endroit différent, l'un sur le "je pense", l'autre sur le "je suis », révélant par là même le paradoxe intime qui l'habite. En élevant le « je pense » au rang de sujet transcendantal, l'idéalisme réflexif a surestimé la valeur du «je pense » au détriment du « je suis». Là, «le cogito tend à l'auto-position ${ }^{17}$, et le vœu qui le consume d'une transparence totale de la conscience à soi-même conduit inévitablement à " une perte d'être ${ }^{18}$, qui rompt les liens de participation " avec ses propres conditions d'enracinement ». Nul doute qu'en faisant jouer Descartes contre lui-même, Ricœur cherche à désamorcer son idéalisme naissant dans le but de libérer le second courant des entraves du premier, découvrant ainsi que le «je suis» est plus large que le "je pense », où celui-ci plonge ses racines, fût-ce par le sentiment confus qu'il a de son union avec le corps : "le "je suis" ou "j'existe" déborde infiniment le "je pense" »19, à commencer par le corps et le cortège de liens involontaires qu'il entraine après lui.

Il y a donc un "faux Cogito » qui est venu faire écran au vrai, et qu'il s'agit de déloger. Autrement dit, la critique que Ricœur puise chez les maîtres du soupçon (Freud (De l'interprétation), Marx (L'idéologie et l'utopie), Nietzsche (Soi-même comme un autre)) n'a jamais eu pour ambition d'éliminer la conscience, mais au contraire d'en extraire plus sûrement encore son noyau apodictique sous le choc des objections capables de détruire les nombreuses idoles qui sont venues l'entourer. Dressant Descartes contre lui-même, dans un geste qui demeure encore cartésien, Ricœur retrouve les deux premières méditations au moment où l'âme, encore inquiète, avant qu'elle ne trouve en Dieu la secrète assurance de son fondement, découvre qu'elle est sans encore savoir ce qu'elle est. Bref, humilier ce qui exalte trop le cogito ne consiste qu'à durcir son noyau apodictique, en rendant le malin génie plus puissant, plus perfide, plus menteur encore qu'il ne l'était, ajoutant à son artifice en lui soufflant des arguments auxquels Descartes n'avait pas pensés et qui furent soulevées après lui. Ce n'est pas même user de la critique contre Descartes que de faire cela, puisque c'est répéter son geste et en user «comme Descartes usait

\footnotetext{
17. P. Ricœur, Philosophie de la volonté, « Le volontaire et l'involontaire », vol. I (cité Ph.V, I), Paris, Seuil, 1950, p. 32.

18. Idem, p. 37.

19. Idem, p. 117.
} 
des arguments sceptiques contre le dogmatisme de la chose $»^{20}$. On s'aperçoit alors que ce sont ces contestations mêmes qui vont permettre à Ricœur de plonger le cogito au milieu d'un langage qui lui livre sa condition originaire.

Parmi tous les instruments critiques dont se servit Ricœur, prenons Heidegger pour exemple. On a souvent lu l'article que Ricœur lui avait consacre ("Heidegger et la question du sujet») comme une objection implicite au Dasein, qui mimait malgré tout le sujet qu'il était censé remplacer. Ce n'est pourtant pas ce que fait Ricœur, qui se sert des armes critiques que lui donne Heidegger pour épurer le sujet dont il se veut, lui, l'héritier : "Il s'agit moins d'une critique du cogito comme tel que d'une critique de la métaphysique qui lui est sousjacente ${ }^{21}$, à savoir le modèle de certitude auquel le cogito " se mesure et se satisfait $»^{22}$. Autrement dit, ce qu'Heidegger a surtout permis, c'est d'évacuer la position idéaliste du cogito à laquelle il croyait pouvoir réduire celui-ci, sans s'apercevoir qu'il n'éliminait qu'une de ses interprétations possibles, qui recevait Descartes depuis Kant, Fichte et Husserl. Le cogito n'est pas une "vérité intemporelle », mais a surgi sur un sol philosophique qui était celui de la science, et qui circulait dans une pré-compréhension de l'être comme être-représenté. À l'âge de la représentation, il devait donc y tenir le rôle de fondement, devenir le "centre auquel l'étant est rapporté $»^{23}$ et devant lequel le monde se dresse comme un tableau - où autoposition du sujet et certitude de l'objet se faisaient pendant. Mais le cogito ne contient-il pas davantage qu'il n'était possible à son époque d'entendre, et qui fut occulté par cela même à quoi on l'avait réduit d'abord ? S'il veut être reçu à notre époque, qui n'est plus celle de Descartes, ne doit-il pas quitter «l'événement sous-jacent à notre culture ", duquel il avait jailli ? Tel est le profit que Ricœur cherche à tirer de la critique heideggérienne : liberer le cogito en vue d'une réappropriation future de ses virtualités inexploitées. Bref, en détruisant le cogito dans sa version épistémologique («je pense ») (« conçu comme un simple principe épistémologique $»^{24}$ ), il $s^{\prime}$ agit de le restituer dans sa version ontologique («je suis»): «le problème ultime de Descartes n'était pas je pense mais je suis, comme d'ailleurs l'atteste la suite des propositions qui, de l'existence de l'ego, procède à $l^{\prime}$ existence de Dieu et à l'existence du monde $»^{25}$. Autrement dit, la destruction du subjectum comme fondement, autorise Ricœur à le sauvegarder comme je. Elle est « la condition d'une juste répétition de

\footnotetext{
${ }^{20}$. P. Ricœur, «Une interprétation philosophique de Freud », C., p. 172.

${ }^{21}$. P. Ricœur, « Heidegger et la question du sujet », C., p. 222.

22. Ibid., p. 224.

23. Ibid., p. 227.

24. Ibid., p. 232.

25. Ibid., p. 225.
} 
la question de $\mathrm{l}^{\prime}$ ego $»^{26}$, qui, en ôtant au cogito le privilège indu de l'immédiateté, creuse une excavation sous le «je pense », d'où jaillit l'être oublié qu'il faut désormais se réapproprier : le "je suis », sitôt qu'il déborde de toutes parts l'abstraction vide du « je pense », ne se rend accessible qu'indirectement et doit devenir pour cette raison même le thème d'une herméneutique, et non plus l'objet d'une intuition. "C'est pourquoi la reprise du "je suis" ne relève pas seulement d'une phénoménologie, au sens d'une description intuitive, mais d'une interprétation, précisément parce que le "je suis" est oublié ${ }^{27}$.

Là se révèle enfin un cogito élevé à sa condition langagière, qui ne peut se connaître qu'en passant par la médiation des signes. Car il n'était pas possible de l'élever à cette hauteur sans l'abaisser d'abord dans sa prétention idéaliste à se poser dans l'être, dans une transparence totale à soi-même. Sitôt qu'il cesse de se prendre "pour le créateur du langage $»^{28}$, le cogito s'attache aux signes sensibles, qui ne s'effacent plus devant leur sens pour lui, et n'accède à ses cogitata qu'en épaississant son vouloir-dire. Il est ainsi lié au sens symbolique, et le tout le lie à lui-même en donnant un contenu à ses pensées, une " chair ", une "densité », qui l'empêchent d'être transparent à soi. Ainsi le noyau apodictique du cogito qui a résisté aux coups de la critique, ré-émerge alors, indubitable, comme sujet parlant plutôt que sujet pensant, plongé au milieu de signes où s'expriment le "je suis ", comme un «désir d'être »: « je suis » sans savoir « qui suis-je »29. Le cogito est un point de départ qui ne commence rien ; il est appelé à retourner à ses sources où le langage était encore naissant. Il arrive plutôt au terme d'une réappropriation de soi par soi à travers les symboles qui nous parviennent par delà les frontières de la philosophie. Par là est fondée la logique du double sens : «Le noyau d'apodicticité du "je pense" est aussi le transcendantal de la fonction symbolique; autrement dit: ce qui est invincible à tout doute, c'est l'acte de recul et de distance qui crée l'écart par lequel le signe est possible et c'est la possibilité d'être relié de façon signifiante, et non pas seulement causale, à toutes choses $\Perp^{30}$. L'herméneutique trouve sa justification dernière, obtenant là « dans la nature même de la pensée réflexive le principe d'une logique $d u$ double sens ${ }^{31}$. Au fond, la logique primordiale du double sens repose sur la dualité de l'être et du pensable, qui permet à Ricœur d'écrire : «Réfléchir et interpréter [1] es symboles sont un seul et même acte $»^{32}$.

\footnotetext{
26. Ibid., p. 228.

27. Ibid., p. 229.

28. Ibid., p. 231.

29. P. Ricœur, «La question du sujet: le défi de la sémiologie », C., p. 262.

30. Ibid., p. 258.

31. P. Ricœur, E., p. 59.

32. Ibid., p. 63.
} 


\section{La mouvance phénoménologique : Husserl et Ricœur - la double intentionnalité du symbole}

Qu'apporte ensuite à Ricœur la phénoménologie, sinon des moyens exceptionnels capables de fouir dans les régions encore inexploitées du Cogito cartésien, en l'arrachant à son mutisme et en l'insérant à l'intérieur du langage ? Aussi Ricœur ne pouvait poursuivre la «ligne » réflexive qu'en se mettant «à l'école de la phénoménologie » et en s'installant dans sa " mouvance». Mais c'est encore dire que la tradition cartésienne reste son ancrage, sa " ligne » fermement maintenue, et qu'il ne trouve dans la phenoménologie husserlienne qu'un puissant relais pour la continuer. En terre cartésienne, Husserl ne peut arriver que second; il ne peut servir à commencer à nouveaux frais, mais à recommencer le geste cartésien de façon plus authentique. Bref, ce qui apparaît comme une hérésie d'un point de vue husserlien doit répondre à une fidélité plus ancienne et plus grande à Descartes.

Aussi que recherche-t-il chez Husserl ? Non pas une doctrine, il en a déjà une, mais une méthode, qui permet de poser le sujet dans le langage même ; c'est ce que veut dire "mouvance ». Dira-t-on qu'il déforme la méthode phénoménologique pour couler en elle une matière étrangère aux prétentions de Husserl ? Mais nombreux des articles qui composent À l'école de la phénoménologie s'emploient à anticiper l'objection et à montrer que la méthode que pratiquait Husserl allait, sans qu'il s'en fût clairement aperçu, en sens contraire de l'interprétation idéaliste où il l'avait réfléchi. Én effet, le travail en acte du phénoménologue ne pouvait qu'entraîner la constitution de la " chose » dans une voie régressive, allant de synthèses actives en synthèses passives, qui repoussait indéfiniment le moment où celle-ci pût se fonder dans l'ego, au point de subvertir «le projet d'autofondation radicale ${ }^{33}$ qui avait d'abord été celui de Husserl. Il le jeta ainsi, comme malgré lui, dans le monde-de-la-vie auquel nous appartenons préalablement avant toute opposition sujet / objet. Citons un passage, qui donne assez bien le geste critique et le ton général à l'égard de Husserl : " Pour ma part, plus je lis Husserl, plus j'avance dans la conviction que la méthode pratiquée tire le philosophe dans un sens de moins en moins compatible avec la méthode interprétée philosophiquement. La méthode pratiquée tend vers " 1 'approfondissement ou la consécration de [l']attitude originelle" d'engagement dans le monde. La méthode interprétée tend vers un idealisme solipsiste qui déleste définitivement la "chose" de son altérité rela-

${ }^{33}$ P. Ricœur, « De l'interprétation », TA, p. 31. 
tive $»^{34}$ en la réduisant «à la vie monadique de l'ego $»^{35}$. Il s'agit alors, pour Ricœur, de retirer à la méthode phénoménologique son soubassement doctrinal idéaliste, comme il lui fallait résister à la tentation idéaliste qui pesait sur l'entreprise cartésienne: "Ce que l'herméneutique a ruiné, ce n'est pas la phénoménologie, mais une de ses interprétations, à savoir son interprétation idéaliste par Husserl luimême $»^{36}$.

Et sitôt qu'on leur ôte l'ambition d'autofondation, toutes les opérations fondamentales de la phénoménologie prennent un tour tout différent, qui s'accorde d'emblée à l'herméneutique parce qu'elles accordent la conscience aux signes dans lesquels elle se perd, se cherche et se retrouve. Pour que la greffe entre elles pût prendre, il ne fallait donc pas d'abord ajouter, mais retrancher. Il suffit que les choses se constituent pour moi, sans plus l'être «en" moi et «à partir » de $\mathrm{moi}^{37}$, et tout devient alors signification, " universelle médiation entre le sujet et le monde ${ }^{38}$. L'intentionnalité est toujours la " grande découverte » de la phénoménologie, mais en toute rigueur cet acte de viser quelque chose, sans pouvoir se viser lui-même qu'à travers le sens visé, pose que "nulle conscience n'est conscience de soi avant d'être conscience de quelque chose ${ }^{39}$, et impose par là la nécessité $\mathrm{d}^{\prime} u$ " retour à soi à partir de son autre ${ }^{40}$. De même, la réduction cesse de rendre tout être relatif à la conscience comme le sens qu'il est pour elle ; une fois coupée de la doctrine idéaliste, elle se contente de nous arracher à notre appartenance primordiale au monde, et laisse ainsi "paraître notre rapport au monde » en le donnant "comme signification à expliciter » ${ }^{41}$. Elle apparaît alors " comme le "transcendantal" du langage ", qui fait de celui-ci, ni un fondement, ni un objet, mais le "médium ", c'est-à-dire la possibilité pour l'homme « de se rapporter au réel en le désignant au moyen des signes $»^{42}$. Bref, la réduction est à l'origine de la fonction symbolique, par quoi l'homme s'ouvre à une vie signifiante ; et la phénoménologie devient elle-même "une théorie du langage généralisé »43. Ricœur suit ainsi son propre chemin dans le mouvement de l'œuvre de Husserl : il s'arrête d'un côté à la neutralité métaphysique souhaitée par les Recherches logiques, suit de l'autre côté l'élargissement que

\footnotetext{
34. P. Ricœur, «Sur la phénoménologie », À l'école de la phénoménologie (cité EP), (1986), Paris, Vrin, 2004, p. 168.

35. P. Ricœur, «Études sur les Méditations cartésiennes de Husserl », EP, p. 230.

36. P. Ricœur, "Phénoménologie et herméneutique : en venant de Husserl... », TA, p. 43.

37. P. Ricœur, «Sur la phénoménologie», EP, p. 184.

38. P. Ricœur, « La question du sujet : le défi de la sémiologie », C, p. 242.

39. P. Ricœur, «Phénoménologie et herméneutique : en venant de Husserl... », TA, p. 63.

40. P. Ricœur, « La question du sujet : le défi de la sémiologie », C, p. 257.

41. Ibid., p. 243.

42. Ibid., p. 253, 252, 254.

43. Ibid., p. 243.
} 
Husserl opéra à partir des Ideen I vers des significations plus larges que la seule signification logique, et l'accompagne enfin dans ses derniers travaux jusqu'à ce qu'elles se récupèrent toutes depuis notre rapport primordial au monde (Lebenswelt) qui ne s'explicite que par elles.

Tout est donc signe. Contre Merleau-Ponty, il n'est pas possible de faire précéder une phénoménologie du langage par une phénoménologie de la perception; elles doivent s'entrelacer dans une « dialectique du signifier et du percevoir, du dire et du voir $»^{44}$. Le sujet dépasse toujours son point de vue fini vers le sens de la chose visée, qu'il ne possède pourtant pas et qui attend du langage d'être porté dans la lumière. Le langage seconde ainsi le corps pour signifier la chose et dire plus que les perspectives limitées sur lesquelles la perception s'arrête. Tout est signe ; mais tout n'est pas symbole. Celui-ci se définit par le double sens. Comment le sujet en vient-il à se comprendre à travers l'opacité des symboles plutôt qu'à l'aide de signes moins équivoques? Il faut en effet comprendre comment le symbole en vient lui-même à s'insérer dans le discours philosophique. Et c'est encore la tâche d'une analyse intentionnelle, inspectant la faillibilité de notre pouvoir de sentir, de connaître et d'agir, que de saisir la nécessité pour l'homme de parler en énigmes, et ainsi de dévoiler l'enracinement phénoménologique de l'herméneutique elle-même. Or il apparaît qu'en se déplaçant du monde vers le sujet qui s'immerge en lui, le langage bute, jusqu'à se briser, sur la double intentionnalité de la conscience, qui vise au sens et à son remplissement, lesquels s'excèdent l'un par l'autre. Certes, quoiqu'il advienne, "je suis double intentionnalité $» 45$, mais cette non-coïncidence que je suis à moi-même est le plus souvent oblitérée dans le face-à-face qui me rassemble et $\mathrm{m}^{\prime}$ oppose aux objets. Visée de perception et visée d'entendement convergent alors. La dualité, quelque soit d'ailleurs la manière de la nommer, celle de la présence et du sens, celle de la sensibilité et de l'entendement, celle du sentir et du connaître, celle du fini et de l'infini, celle du Voir et du Dire, s'amortit en effet dans la synthèse de l'objet, grâce à un troisième terme qui s'épuise à les réconcilier, sans pouvoir se réfléchir pour soi. Telle est pour Ricœur l'imagination transcendantale de Kant, " art caché dans les profondeurs de l'âme humaine $» 46$. Mais si cette dualité originaire se cache dans l'objet, elle se dramatise dans le sujet; elle se révèle à lui comme disproportion, s'éprouve comme misère et l'oblige à s'atteindre dans un langage indirect. Autrement dit, ce sont des « schèmes » qui manquent pour se penser soi-même. Et comme tout notre langage s'est élabore dans la dimension de l'objectivité, le langage qui portera sur

44. P. Ricœur, Ph. V. II, p. 64.

45. Ibid., p. 66.

46. Ibid., p. 80 ; Kant, Critique de la raison pure, A 141. 
la subjectivité sera essentiellement symbolique, en disant toujours plus et autre chose que ce qu'il dit, allant du sens au sens, s'excédant lui-même dans un mouvement d'amplification et d'intériorisation, qui nous assimile au symbolisé.

En effet, le signe, quand il est technique, ne dit que ce que le sujet veut qu'il dise, d'un lien conventionnel. Le symbole, lui, est un signe non technique et veut dire autre chose que ce qu'il dit, d'un lien analogique. Le premier est idéalement univoque, peut s'absenter de la chose même et signifier à vide, et rend possible un langage formalisé qui se substitue au monde des choses pour en faire plus grand usage. Le second est équivoque, et implique au sein même de sa visée une double intentionnalité qui n'emporte le sujet d'un sens premier, littéral, vers un sens second, figuré, que si celui-ci vit et s'installe dans la présence de ce qu'il vise, ou il trouve la matière analogique d'un nouveau remplissement. Par exemple, le souillé veut dire littéralement la tâche physique, mais est habité par un autre signifié, la souillure existentielle, qui se donne en lui comme une tâche, laquelle «vise une certaine situation de l'homme dans le sacré qui est précisément l'être souillé, impur $\gg^{47}$. C'est par le second sens que s'opère un retournement où, au lieu que l'homme parle à l'aide de signes, le langage parle à l'homme, s'adresse à lui dans une sorte de "contreintentionnalité »(Levinas), qui est une "inversion de la visée intentionnelle $»^{48}$ (Ricœur). Le sujet se découvre alors interpellé par le symbole. Il prélève ainsi dans le monde des symboles de lui-même qu'il s'approprie (symbolisme cosmique), intériorise en lui (symbolisme onirique), afin de renouer avec l'expérience vive du langage (symbolisme poétique).

\section{La variante herméneutique, et le retour à une réflexion concrète - « le symbole donne à penser»}

Se déprendre du cogito immédiat, c'est libérer le langage qui le précède, se réinstaller dans sa plénitude, et déployer l'architecture du symbole comme double sens, ou multiple sens, avec pour rôle à chaque fois de montrer en cachant: "Une méditation sur les symboles part du plein du langage et du sens toujours déjà là ; elle part du milieu du langage qui a dejà eu lieu et où tout a déjà été dit d'une certaine façon ${ }^{49}$. Le cogito est d'emblée médiatisé par le langage et ne pourra reconquérir son désir d'être qu'en explorant les symboles qui

\footnotetext{
47. Ibid., p. 218.

48. P. Ricœur, E., p. 40.

49. P. Ricœur, « Herméneutique des symboles et réflexion philosophique (I) », C., p. 283.
} 
l'entourent, par restauration et réinvention de leur sens. Tel est le mouvement qui conduit Ricœur jusqu'au niveau proprement linguistique et le fait opter pour l'herméneutique, qui l'empêche de clore l'univers des signes sur lui-même en un système de signifiants / signifiés qui exclurait le rapport à la chose, qui le prévient par là même du risque d'idéalisme linguistique : le symbolique n'est pas un monde à lui-même, mais "un milieu d'expression pour une réalité extra-linguistique », qui pose le Cogito dans l'être et situe l'interprétation «à la charnière $[\ldots$.$] du langage et de l'expérience vécue »^{50}$. C'est là que l'herméneutique trouve son sol le plus fertile pour déployer le problème du sens, offrant au concept d'interprétation la même extension que le symbole, comme détecteur d'un sens caché dans le sens apparent.

Je nais en ayant oublié qui je suis, et tentant de rassembler mes membres épars, je déchiffre les signes qui ont été disséminés dans l'espace et dans le temps, dont j'évoque la mémoire et que je m'efforce à nouveau d'entendre. Mais il faut alors plus qu'une phénoménologie des symboles, simple instrument d'écoute qui découvre leur pouvoir révélant en investissant toutes les cultures au même titre, sans croire ni s'engager dans aucune. Il faut une herméneutique. Et l'herméneutique "proprement dite » doit «quitter la position, ou pour mieux dire 1'exil, du spectateur lointain et désintéressé $»^{51}$. Elle doit lever l'épochè phénoménologique et choisir sa mémoire. Or dans quelle mémoire puis-je me recueillir en puisant dans sa réserve sinon dans le fonds proto-hélénique, sémitique et indo-européen dont j'ai hérité ? Ainsi s'amorce le cercle herméneutique qui consent à s'enfoncer dans la contingence des cultures dans lesquelles nous circulons et qui font notre situation dans l'univers des symboles : "Croire pour comprendre et comprendre pour croire $»^{52}$. Là où les Anciens croyaient et comprenaient d'un même mouvement, dans une naïveté première, nous autres modernes ne pouvons revivre « les grandes symboliques du Sacré » $\mathrm{qu}^{\prime}$ « en interprétant », dans « une seconde naïveté $»^{53}:$ "Croire pour comprendre», car notre cœur nous convainc que là réside le chiffre de notre être ; "comprendre pour croire », car nous en sommes aujourd'hui séparés et qu'il en faut la clef. C'est alors en entrant dans le cercle que la distance se surmontera peu à peu et que la philosophie pourra se revivifier « au contact des symboles fondamentaux de la conscience ${ }^{54}$.

\footnotetext{
${ }^{50}$. P. Ricœur, «Le problème du double-sens comme problème herméneutique et comme problème sémantique $», C$., p. 67.

51. P. Ricœur, « Herméneutique des symboles et réflexion philosophique (I) », C., p. 294.

52. P. Ricœur, E., p. 548 ; Ph. V. II, p. 570 ; C., p. 294.

53. P. Ricœur, Ph. V. II, p. 570.

54. Ibid.
} 
Mais il suffit d'empêcher l'univocité et dire "deux » (équivocité) pour dire aussitôt " plusieurs » (plurivocité) et être entraîné dans la vie des symboles, qui se fondent les uns dans les autres, se reprennent et se renversent, se dépassent et se retiennent, et s'intériorisent à mesure qu'ils avancent. Sans cette dynamique des symboles, ceux-ci ne seraient qu'images, et rien de plus. Ainsi "souillure, péché, culpabilité » flèchent le parcours d'un « devenir soi » dans l'histoire de la conscience, où chaque figure revient, plus pure, dans les suivantes qui l'avaient d'abord niée - replaçant l'ensemble dans une " dialectique $\gg^{55}$ qui suit Hegel implicitement ici, explicitement dans l'Essai sur Freud. L'expérience vécue de la faute s'avive, se nuance, se purifie et $s^{\prime}$ enrichit du langage symbolique qui l'articule en médiatisant notre rapport à soi. Dans la première partie de la Symbolique du mal, Ricœur montre en effet comment l'ancien symbolisme de la souillure craque sous la poussée d'un nouveau symbolisme, celui du péché, l'image de l'écart et de la déviance renversant celle du contact extérieur, et comment celui-ci à son tour est dépassé de l'intérieur par le symbolisme de la culpabilité qui promeut enfin la «conscience » et intériorise complètement le moment de la faute afin de l'entendre comme un mauvais usage de la liberté. C'est alors que les symboles de la souillure et du peché, tournés vers l'intériorité, s'adressent à nous, décollent de leur sens littéral, qui leur était attribué par le contexte historique initial, et se manifestent comme symboles purs pour dire notre "serf-arbitre $»^{56}$. Si la seconde partie du livre accompagne ensuite ces symboles primaires dans leur reprise au sein d'ensembles plus vastes que sont les mythes, il apparaît déjà que le mouvement des symboles est en lui-même une invitation à la réflexion. À la fin, on dira que chaque symbole n'aura épuisé son sens et ne sera pur que lorsqu'il aura cessé d'être "image » et révèlera le sens figuré qu'il masquait auparavant, offrant à la réflexion des "schèmes » à partir desquels une pensée autonome par " concepts » peut s'édifier. Tel est le tryptique kantien qu'une réflexion sur les symboles doit traverser : intuition / schème / concept.

Car après que la réflexion a appelé l'herméneutique, l'herméneutique appelle désormais la réflexion, sans quoi elle ne serait pas même philosophique. C'est qu'il y a bien des manières de parcourir la pluralité des sens, et sans l'arbitrage philosophique qui les soumet à l'épreuve de la compréhension de soi, les hermeneutiques rivales se livreraient à un conflit indépassable, qui ferait songer au champ de bataille de l'ancienne métaphysique. Ricœur rencontre frontalement un tel conflit entre l'herméneutique restauratrice et progressive inspirée de Hegel, déjà évoquée, et l'herméneutique réductrice et régres-

55. Ibid., p. 307.
56. Ibid., p. 362. 
sive qu'il découvre dans la psychanalyse freudienne. Si l'une et l'autre s'enchevêtrent dans la trame du double sens, elles interprètent le même symbole dans deux directions opposées, en avant et en arrière. La première est soucieuse de promouvoir ce que le symbole dévoile, c'est-à-dire la "diversité qualitative des visées et des intentions ${ }^{57}$ (téléologie), alors que la seconde se tourne vers ce que le symbole masque encore, c'est-à-dire l'hylétique en tant qu'elle est toujours la même, simple résurgence de notre enfance (archéologie). Reposant sur l'ambiguité de sa fonction (montrer/cacher), ce n'est que dans le symbole lui-même que la réflexion concrète pourra réconcilier les interprétations rivales, comme étant l'archéologie et la téléologie d'un sujet: "Seul a une arché un sujet qui a un telos » ${ }^{58}$.

Ainsi se fonde l'espoir de reprendre la réflexion philosophique sur un fonds qui n'est pourtant pas à elle, et qu'une formule kantienne récapitulait aux yeux de Ricœur : "Le symbole donne à penser ${ }^{59}$. $\mathrm{D}^{\prime}$ abord, le symbole est un don ; "il est donnant, parce qu'il est une intentionnalité primaire qui donne le sens second $»^{60}$. Il est le "don du langage ", un don de sens, d'une "abondance de sens, dont il dispose si peu que c'est elle qui lui donne à penser ${ }^{61}$. Donne-t-il même plusieurs sens? Alors loin d'empêcher la réflexion, il la stimule davantage et fait du langage une "fête", où se retrouve "l'impulsion du dire sans fin » ${ }^{62}$. Il devance le sujet, l'entraîne dans son mouvement, lui indique la direction et l'oblige à penser ce qui le précède et le fonde (archéologie). Ensuite, « ce que le symbole donne, $c^{\prime}$ est à penser. À partir de la donation, la position $»^{63}$. Il est un « appel

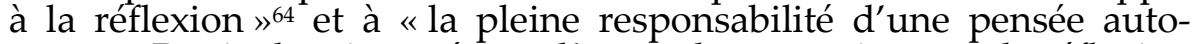
nome ». Partie des signes épars d'une culture contingente, la réflexion doit ainsi remonter progressivement à l'universalité et la nécessité, qui ont toujours été la prétention de la philosophie. Cessant de penser

\footnotetext{
57. P. Ricœur, E., p. 543.

58. Ibid., p. 481.

${ }^{59}$. P. Ricœur, Ph. V. II, p. 566 sq. ; encore que Kant, dans la citation véritable, continue d'entendre le symbole comme infra-conceptuel : « Par l'expression Idée esthétique j'entends cette représentation de l'imagination, qui donne beaucoup à penser, sans qu'aucune pensée déterminée, c'est-à-dire de concept, puisse lui être adéquate et que par conséquent aucune langue ne peut complètement exprimer et rendre intelligible " (Critique de la faculté de juger, tr. fr. A. Philonenko, Paris, Vrin, 1993, § 49, p. 213. Cf. sur leur différence J. L. Vieillard-Baron, « la spécificité du symbolique dans la sphère religieuse ", Laval théologique et philosophique, vol. 52, n 2, 1996, p. 411-424.

${ }^{60}$. P. Ricœur, « Herméneutique des symboles et réflexion philosophique (I) », C., p. 286.

61. P. Ricœur, E., p. 48, 59.

62. P. Ricœur, «Le problème du double-sens comme problème herméneutique et comme problème sémantique », C., p. 95.

63. P. Ricœur, Ph. V. II, p. 568.

64. P. Ricœur, E., p. 48.
} 
«dans les symboles » pour "penser à partir des symboles »65, le cogito a pour tâche de se poser dans l'être (téléologie). Assurément, l'herméneutique des symboles ne sert qu'à désigner la première philosophie de Ricœur, qui deviendra à ses propres yeux une simple étape vers l'herméneutique du texte, qui offrait une base plus large où l'accueillir. Mais déjà se noue dans la dialectique vivante du symbole concret une herméneutique du soi, qui ouvre pour sa philosophie de la personne des ressources nouvelles et des dimensions inédites en la rechargeant dans le langage : d'où venons-nous ? que sommes-nous? où allons-nous? Par le symbole, le sens devance le sujet (archéologie) et l'envoie au-devant de lui-même (téléologie), le situe au sein de la totalité de l'être auquel il participe. À travers son interprétation créatrice, il augmente sa compréhension de lui-même.

65. P. Ricœur, Ph. V. II, p. 574. 